\section{Vagus nerve pain referred to the craniofacial region. A case report and literature review with impli- cations for referred cardiac pain}

\author{
D. E. Myers ${ }^{1}$
}

IN BRIEF

- Neck, tooth and jaw pain can occur with or without chest pain in angina pectoris and myocardial infarction.

- The mechanism of this referred pain has not been established and is puzzling.

- Stimulation of a branch of the left vagus nerve from the heart can cause tooth. neck and jaw pain.

- This mechanism can help the dental provider to explain the cardiac significance of activity related jaw pain to patients.

The pain of angina pectoris and myocardial infarction is sometimes referred to the head and neck region. The mechanism for this effect remains obscure. A case is presented here that reports that electrical stimulation of a cardiac branch of the left vagus nerve in humans can cause referred craniofacial pain. This leads to the hypothesis that the vagus nerve plays a role in mediating this pain. A review of the clinical and physiologic literature supports this hypothesis.

\section{INTRODUCTION}

Referral of pain from sites within the head and neck region is a common occurrence in dental practice. While much less common, pain can be referred to the craniofacial area from the heart in cases of angina pectoris and myocardial infarction. Typical ischaemic coronary pain is an uncomfortable sensation in the centre of the chest. It has been known for many years that this pain may 'radiate' to the neck, shoulder, jaw, back or arm. Nonetheless, the full extent of the craniofacial pain of coronary ischaemia has only recently been appreciated..$^{1-3} \mathrm{In}$ one study, ${ }^{3}$ a total of $6 \%$ of subjects had craniofacial pain as the only complaint of coronary ischaemia while an additional 32\% experienced craniofacial pain concomitant with pain in other regions. However, the mechanism of the referred pain from the heart to the craniofacial area remains obscure. A case is presented here which suggests that stimulation of the thoracic cardiac branch of the left vagus nerve can produce pain perceived as originating from the left sided throat,

\footnotetext{
'Department of Oral Diagnosis, Dental Associates, Ltd. Of Wisconsin, 11711 W. Burleigh St., Wauwatosa, WI 53222, USA

Correspondence to: Dr Daniel Myers

Email:dnaanddan@yahoo.com
}

\section{Refereed Paper}

Accepted 21 November 2007

DOI: $10.1038 /$ bdj.2008.101

${ }^{\oplus}$ British Dental Journal 2008; 204: 187-189 neck, jaw and teeth. This leads to the hypothesis that the vagus nerve mediates the referral of pain to the ipsilateral craniofacial region in angina pectoris and other medical conditions. A review of the clinical and physiologic literature provide support for this concept.

\section{CASE REPORT}

A 63-year-old female with a history of Graves' Disease and chronic depression presented to the Oral Diagnosis Department of a large group dental practice with a complaint of a left sided toothache that had been present for nearly two weeks. Her current medications included synthroid, premarin, fluoxetine, clonazepam, lamotrigine, olanzapine and zolpidem. The patient reported that the tooth was not sensitive to thermal stimuli or chewing. Radiographic examination, inspection, percussion and palpation of the lower and upper left dental quadrants showed no positive findings. Tooth 18, where the patient felt her pain, had a previous endodontic procedure that appeared to be stable. Tooth 19 had been extracted years earlier and was replaced by a fixed bridge from 18 to 20 . The patient believed that the pain coincided with the frequency and duration of the vagus nerve stimulator (VNS) (Cyberonics, Inc., Houston, TX, USA) that had been implanted the previous week as part of an experimental study to determine its efficacy in the management of intractable depression. VNS is also used in the treatment of drugresistant epilepsy. The device includes a generator implanted in the chest and a stimulating lead electrode wire attached the left vagus nerve in the neck. The cycle in this case as per usual was a 30 second stimulation period at five minute intervals. Stimulus intensity was $2.0 \mathrm{ma}$, frequency was $20 \mathrm{HZ}$ and pulse width was $250 \mathrm{~ms}$.

The episodes of pain were intermittent, coinciding with the duration (30 seconds) and frequency of stimulation (every five minutes). I verified this with my wristwatch. However, the patient reported that sometimes a period of stimulation would occur without any experience of pain. At other times the pain would outlast the stimulation. I informed the psychiatrist of the situation and he made several attempts to adjust the stimulation parameters. My tentative diagnosis at time of first presentation was referred pain emanating from the area of stimulation. When my patient asked me to explain the possible mechanism of this effect I stated simply that sometimes the pain of heart disease can be felt in the jaw and teeth. I wondered, was this the same phenomenon?

\section{LITERATURE INVESTIGATION}

The first question I had was what is the anatomical site where the vagus stimulation occurs? Based on the guidelines of the VNS manufacturer, electrodes are placed on the proximal end of the 
thoracic cardiac branch of the vagus nerve in the carotid sheath in the neck (Fig. 1). According to Gray's Anatomy ${ }^{4}$ the thoracic cardiac branch of the vagus nerve carries visceral afferent input from the heart and great vessels. The second question I had was have others reported similar findings? A Medline search was conducted to determine if other authors have reported craniofacial pain as a side effect of VNS and, if so, what sites are most commonly reported? In general VNS side effects are uncommon and mild. Nonetheless both pain and non-painful conditions such as hoarseness, coughing, dyspnea and paraesthesia around the stimulation site have been reported. Severity of the side effects may be proportional to the pulse width, current amplitude, frequency and duration of stimulation. ${ }^{5}$ These can be easily adjusted by the managing physician. Painful side effects of VNS were reported in nine studies ${ }^{5-13}$ (Table 1). The most common site of pain reported was the throat followed by the jaw, neck, teeth and ear. Neither chest nor arm pain was reported. In one article, jaw, tooth and ear pain were the only side effects in the series of three cases. None of the reports discussed the mechanism of craniofacial pain vis-áa-vis referred cardiac pain. My final question was is there any physiologic evidence to support the hypothesis that vagal afferents from the heart could cause jaw and tooth pain? Research in rodents ${ }^{14}$ and primates ${ }^{15}$ found that noxious electrical and chemical stimulation of cardiac branches of the left vagus nerve caused activation of left spinothalamic tract cells at the level of the trigeminothalamic tract. This provides a possible physiologic basis for referral of cardiac pain to the trigeminal region.

\section{COMMENT}

Both the clinical and physiological literature lend support to the notion that the vagus nerve mediates the throat, neck, and jaw pain associated with coronary ischaemia. The correlation of the frequency and duration of pain with those of electrical stimulation in the case report presented provide strong support for the idea that the stimulation caused the pain. In this same

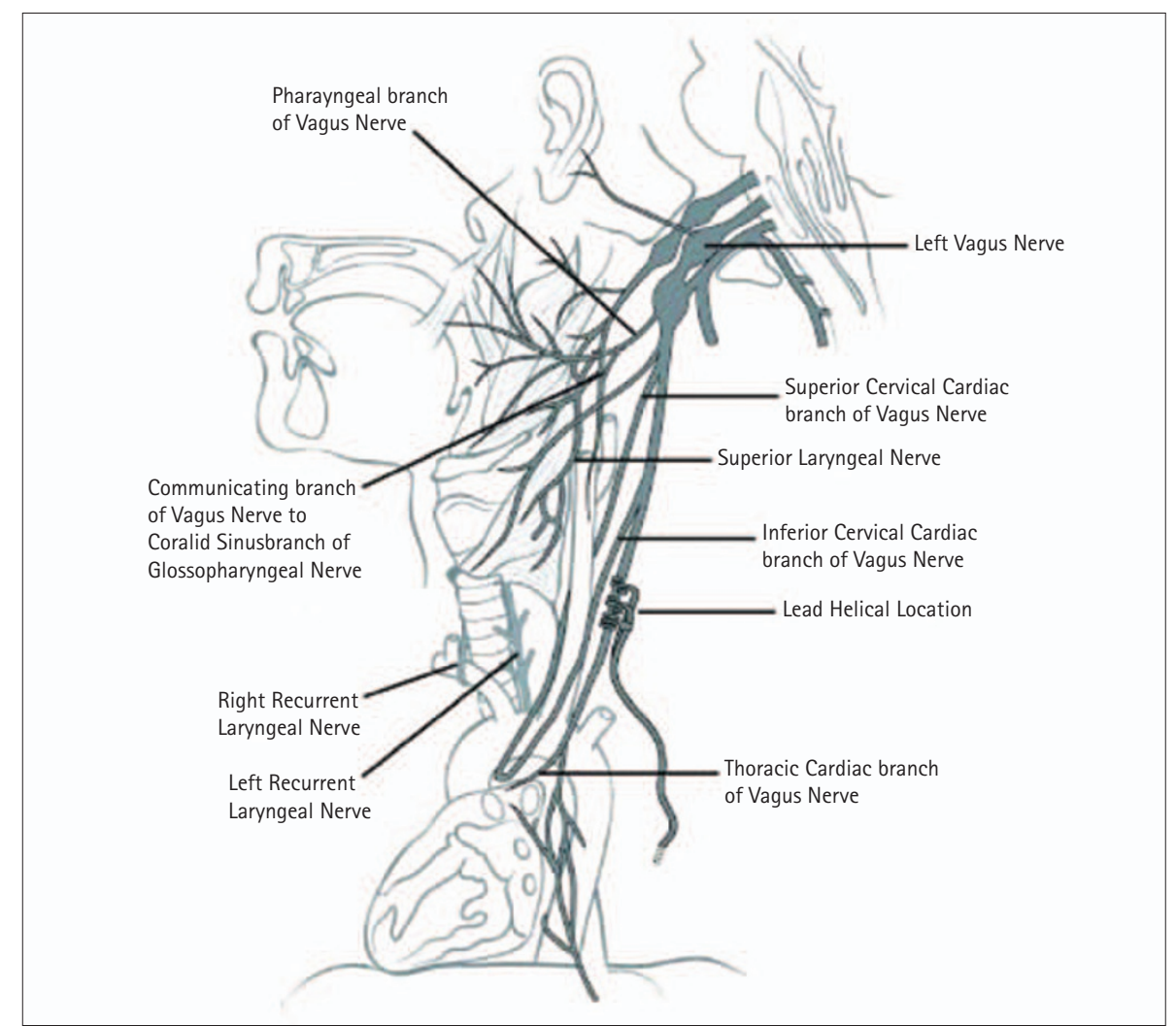

Fig. 1 Anatomy of the vagus nerve showing the location of the stimulating electrode lead (by permission, Cyberonics, Inc)

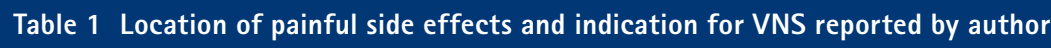

\begin{tabular}{|c|c|c|c|c|c|c|}
\hline First Author & Throat & Neck & Jaw & Teeth & Ear & $\begin{array}{l}\text { INDICATION } \\
\text { E = Epilepsy }\end{array}$ \\
\hline Liporace & $x$ & $x$ & $x$ & $x$ & & $E$ \\
\hline Rush & $x$ & $x$ & & & & D \\
\hline Uthman & $x$ & & $x$ & & & $E$ \\
\hline Handforth & $x$ & & & & & $E$ \\
\hline Ben-Menachem & $X$ & & & & & $E$ \\
\hline DeGiorgio & $x$ & & & & & $E$ \\
\hline Carius & & $x$ & $x$ & $x$ & $x$ & $E$ \\
\hline Shih & $X$ & & X (Cheek) & & & $E$ \\
\hline Kersing & $x$ & & & & & $E$ \\
\hline Total & 8 & 3 & 4 & 2 & 1 & $8 \mathrm{E} 1 \mathrm{D}$ \\
\hline
\end{tabular}

case, the managing psychiatrist firmly believed that the pain was caused by the stimulation.

Neither the human nor the animal model is ideal. However, the recent introduction of VNS has provided information about the vagus nerve in humans that could not be obtained previously due to ethical considerations. The study is limited by its retrospective nature.
On the other hand, none of the patients nor any of the investigators in the cited literature was testing any hypothesis so blinding was not an issue. Despite the relatively small number of patients reporting painful side effects, it is clear that such pain is expected to be present in a percentage of cases. It is not surprising that such pain occurs infrequently in that adjustment of stimulation 
parameters is routinely performed after initiating VNS in order to minimise these effects. I informed the psychiatrist of the situation and he made several attempts to adjust the stimulation parameters. It was a slow process over several months where current parameters were reduced and then elevated to find a level that would be therapeutic yet pain free. At last report, the patient was doing well with the following parameters: intensity $1.5 \mathrm{ma}$, frequency $30 \mathrm{HZ}$, pulse width $130 \mathrm{~ms}$, duration 30 seconds and frequency $1 / 1.8$ minutes.

I must caution that this report focuses only on the role of the thoracic cardiac branch of the left vagus. Other branches of the left vagus, branches of the right vagus, and sympathetic afferents are all likely to play some role in cardiac pain.
It is also possible that other branches of the vagus nerve refer pain to the jaw and teeth.

1. Tzukert A, Hasin Y, Sharav Y. Orofacial pain of cardiac origin. Oral Surg Oral Med Oral Pathol 1981 51: 484-486.

2. Kreiner $M$, Okeson, J.P. Toothache of cardiac origin. J Orofac Pain 1999; 13: 201-207.

3. Kreiner M, Okeson J P, Michelis V, Lujambio M, Isberg A. Craniofacial pain as the sole symptom of cardiac ischemia: a prospective multicenter study. J Am Dent Assoc 2007; 138: 174-179.

4. Gray H. Gray's Anatomy, 29th ed. pp 940-941. Philadelphia: Lea and Febiger, 1973.

5. Liporace J, Hucko D, Morrow R, Barolat G et al. Vagal nerve stimulation: Adjustments to reduce painful side effects. Neurology 2001; 57: 885-886.

6. Rush A J, Marangell L B, Sackeim H A et al. Vagus nerve stimulation for treatment-resistant depression: a randomized, controlled acute phase trial. Biol Psychiatry 2005; 58: 347-354.

7. Uthman B M, Reich A M, Dean J C. Eisenschenk S et al. Effectiveness of vagus nerve stimulation in epilepsy patients: a 12 year observation. Neurology 2004; 63: 1124-1126.

8. Handforth A, DeGiorgio C M, Schachter S C et al. Vagus nerve stimulation therapy for partial-onset seizures: a randomized active-control trial. Neurology 1998; 51: 48-55.

9. Ben-Menachem E Vagus nerve stimulation for the treatment of epilepsy. Lancet Neuro/ 2002; 1: 477-482.

10. DeGiorgio C, Heck, Bunch S. Britton J et al. Vagal nerve stimulation for epilepsy: randomized comparison of three stimulation paradigms. Neurology 2005; 65: 317-319.

11. Carius A, Schulze-Bonhage A. Trigeminal pain under vagus nerve stimulation. Pain 2005; 118: $271-273$

12. Shih J J, Devier D, Behr A. Late onset laryngeal and facial pain in previously asymptomatic vagus nerve stimulation patients. Neurology 2003; 60: 1214 .

13. Kersing $W$, Dejonckere $P H$, van der $A a H E$, Buschman H P J. Laryngeal and vocal changes during vagus nerve stimulation in epileptic patients. J Voice 2002; 16: 251-257.

14. Qin C, Chandler M J, Miller K E, Foreman R D. Responses and afferent pathways of superficial and deeper C1-C2 spinal cells to intrapericardial algogenic chemicals in rats. J Neurophysiol 2001; 185: 1532-1532.

15. Chandler M J, Zhang J, Qin C, Yuan Y, Foreman R D. Intrapericardiac injections of algogenic chemicals excite primate $\mathrm{C} 1-\mathrm{C} 2$ spinothalamic tract neurons. Am J Physiol Regul Integr Comp Physiol 2000: 279: R560-R568. 